\title{
FT-IR spectra and vibrational spectroscopy of Andrographolide
}

\author{
P.K. Singh ${ }^{\text {a }}$, Tanveer Hasan ${ }^{\mathrm{a}}$, Onkar Prasad ${ }^{\mathrm{a}}$, Leena Sinha ${ }^{\mathrm{b}}$, Kanwal Raj ${ }^{\mathrm{c}}$ and \\ Neeraj Misra ${ }^{a, *}$ \\ ${ }^{a}$ Department of Physics, Lucknow University, 226007, India \\ ${ }^{\mathrm{b}}$ Department of Physics, Mahila PG College, Aminabad, Lucknow, India \\ ${ }^{\mathrm{c}}$ Medicinal and Process Chemistry Division, Central Drug Research Institute, Lucknow, 226001, India
}

\begin{abstract}
A complete normal coordinate analysis was performed for andrographolide in terms of the calculation by using Wilson's G-F matrix method and Urey Bradley force field. Normal coordinate analysis has been carried out to understand the dynamical behaviour of the bioactive labdane diterpenoid of the medicinally useful terrestrial plant Andrographis paniculata. The structural elucidation of a novel, biologically active natural product followed by its total synthesis lays the groundwork for investigations into its biological mechanism of action including putative cellular receptor isolation.
\end{abstract}

Keywords: FT-IR spectra, diterpene, normal coordinate analysis

\section{Introduction}

Natural products hold great potential as probes of the cellular processes in which they interfere. Structural and conformational studies lead to hypothesis concerning target ligand interactions while synthesis is a means to test these hypotheses by eventual total synthesis of derivatives. Andrographis has long been used in traditional Indian and Chinese herbal medicine. The most common reported uses were for digestive problems (as is the case with most non toxic bitter herbs such as andrographis) snake bite, and infections ranging from malaria to dysentery [1,2]. Interestingly some of these uses have been validated by modern scientific research. The major constituents in andrographis are diterpene lactones known as andrographolide. These bitter constituent are believed to have immune-stimulating, anti inflammatory, fertility decreasing, liver protective and bile secretion-stimulating actions [3]. Though some older studies suggested andrographis was antibacterial, modern research has been unable to confirm this finding [4]. Although, a number of studies on the structure and stability of andrographolide have been carried out [5-9]. To the best of our knowledge no study on the normal coordinate analysis on andrographolide has been reported so far in the literature.

As a part of our ongoing research work [10-17] on vibrational analysis, phonon dispersion, phase transition in a variety of macromolecules, here in the present communication we report the dynamical study carried out on the diterpene Andrographolide. The purpose of this study assumes importance because of the further work on andrographolide ( i.e., oxidation studies on Andrographolide) [18], in

\footnotetext{
${ }^{*}$ Corresponding author: Neeraj Misra, Department of Physics, Lucknow University, 226007, India. E-mail: misraneeraj@ gmail.com
} 
which presumably some modification by chemical reaction such as oxidation/reduction on poly functional bioactive natural product will generate new reactive site in the molecule, which can be exploited for elaborating and appending pharmacophores to generate patentable compounds of biological interest.

\section{Theory}

The well known Wilson's G-F Matrix method [19-21] with Urey Bradley [22] force field has been used to evaluate the normal modes.

These are given by the eigen values $\lambda$ of the secular equation.

$$
\mathrm{GFL}=\lambda L \quad \text { as } \quad \lambda=4 \pi^{2} c^{2} v^{2} .
$$

The potential is represented as

$$
\begin{aligned}
V= & \sum_{j k} K_{j k}^{\prime} r_{j k}\left(\Delta r_{j k}\right)+\frac{1}{2} K_{j k}\left(\Delta r_{j k}\right)^{2}+\sum_{i j k} H_{i j k}^{\prime} r_{i j} r_{j k}\left(\Delta \phi_{i j k}\right)+\frac{1}{2} H_{i j k} r_{i j} r_{j k}\left(\Delta \phi_{i j k}\right)^{2} \\
& +\sum_{i j k} F_{i k}^{\prime} q_{i k}\left(\Delta q_{i k}\right)+\frac{1}{2} F_{i k}\left(\Delta q_{i k}\right)^{2}+\sum_{i} K_{j}^{w}\left(\Delta w_{j}\right)^{2}+\sum_{j} K_{j}^{t}\left(\Delta t_{j}\right)^{2}
\end{aligned}
$$

where $\Delta r_{j k}, \Delta \phi_{i j k}, \Delta w_{j}$ and $\Delta t_{j}$ are the internal coordinate changes corresponding to bond stretch, angle bend, out of plane deformation and torsion respectively.

The potential energy distribution in the $j$ th internal coordinate for the $i$ th normal mode is given by

$$
(\mathrm{PED})=\frac{L_{j i}^{*} L_{j i} F_{j i}}{\lambda_{i}}
$$

\section{Experimental}

The FTIR spectra of andrographolide have been recorded in CsI on a Perkin Elmer 1800 spectrophotometer. Spectroscopic preparation of sample were carried out under an atmosphere of prepurified nitrogen.Andrographolide was isolated from fresh plant material of Andrographis paniculata to the procedure reported in literature [23]. The compound was identified by comparison with its IR, MS, NMR data with that of reported in literature [22,24].

\section{Results and Discussion}

\subsection{Structure}

The crystal structure of Andrographolide has been reported by several workers [24,25] but the present structure is more accurately determined. The six membered rings are in chair conformation. The furan ring is slightly puckered. The molecule crystallizes in space group $\mathrm{P} 2{ }_{1}$ with the monoclinic unit cell. Basak et al. [26,27] have shown that andrographolide could be considered as an important precursor structure that might require further structural modification for enhancement of its enzyme inactivation 


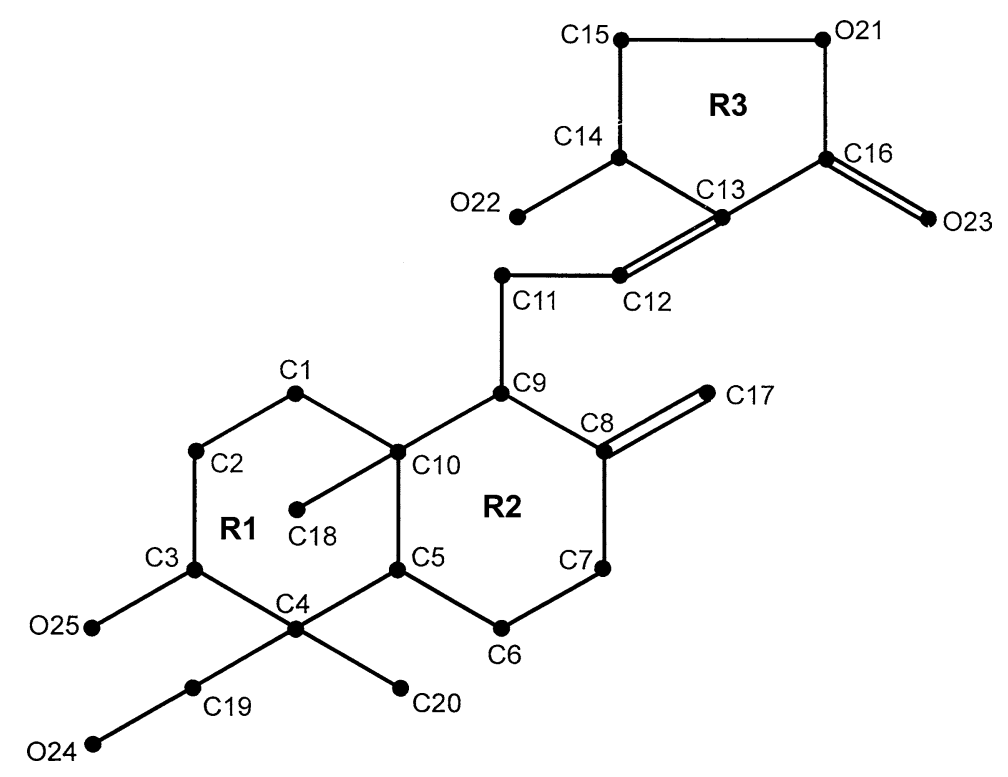

Fig. 1. Model molecular structure of Andrographolide.

property. The modification of hydroxy groups in Andrographis paniculata could lead to a better understanding of its inhibitory action. The actual mechanism by which these diterpenes exert their inhibitory action against convertases is not currently understood but these molecules do contain a highly reactive five membered lactone ring. In andrographolide, the entire structure is essential for biological action and presumably the vibrational modes involving the sites with substantial potential energy contribution play a decisive role in the receptor binding. This justifies the need for an in depth study of the conformation and dynamics of andrographolide.

\subsection{Vibrational Investigations}

Normal coordinate calculations were performed using the program developed by Shimanouchi [28] following the G-F matrix method according to Wilson et al. This method describes the motion in terms of the internal coordinates, which are changes in bond lengths, bond angles and those out of plane bending and dihedral angles. The force constants in terms of these coordinates can be easily visualized and have a physical meaning. We have used a Urey Bradley force field in our calculations. It incorporates intra unit interactions and interactions due to the neighboring units, in addition to the bonded interactions. It also includes the interactions between non-bonded atoms. Force constants were initially taken from the literature [19] and later modified to give the "best fit" with the observed FT-IR spectra.

The andrographolide has 55 atoms, however to reduce the problem to manageable dimensions $\mathrm{CH}$, $\mathrm{CH}_{2}, \mathrm{CH}_{3}$ have been treated as mass points with a mass of 13,14 and 15 respectively. This does not in any way disturb the accuracy of the results reported here. This is because the frequencies belonging to these can be well designated as group frequencies and many of them being in the higher range do not mix with other modes. With this approximation the andrographolide problem reduces to 25 atom problem with 69 normal modes of vibrations. The structural data used for Andrographolide is reported by Spek et al. [29]. The model molecular structure of andrographolide used in normal coordinate analysis is given in Fig. 1. The internal coordinates and force constants for Andrographolide are given in Table 1. The final 
Table 1

Internal co-ordinates and urey bradley force constants $\left(\mathrm{md} / \mathrm{A}^{0}\right)$

\begin{tabular}{|c|c|c|c|}
\hline Internal coordinate & Force constant & Internal coordinate & Force constant \\
\hline 1. $\nu(\mathrm{C} 1-\mathrm{C} 2)$ & 3.531 & 47. $\phi(\mathrm{C} 8-\mathrm{C} 9-\mathrm{C} 10)$ & $1.850(1.080)$ \\
\hline 2. $\nu(\mathrm{C} 1-\mathrm{C} 10)$ & 3.531 & 48. $\phi(\mathrm{C} 8-\mathrm{C} 9-\mathrm{C} 11)$ & $0.480(0.250)$ \\
\hline 3. $\nu(\mathrm{C} 2-\mathrm{C} 3)$ & 3.531 & 49. $\phi(\mathrm{C} 10-\mathrm{C} 9-\mathrm{C} 11)$ & $0.480(0.250)$ \\
\hline 4. $\nu(\mathrm{C} 3-\mathrm{C} 4)$ & 3.531 & 50. $\phi(\mathrm{C} 1-\mathrm{C} 10-\mathrm{C} 5)$ & $1.520(0.980)$ \\
\hline 5. $\nu(\mathrm{C} 3-\mathrm{O} 25)$ & 4.900 & 51. $\phi(\mathrm{C} 1-\mathrm{C} 10-\mathrm{C} 9)$ & $0.480(0.250)$ \\
\hline 6. $\nu(\mathrm{C} 4-\mathrm{C} 5)$ & 3.531 & 52. $\phi(\mathrm{C} 1-\mathrm{C} 10-\mathrm{C} 18)$ & $0.480(0.250)$ \\
\hline 7. $\nu(\mathrm{C} 4-\mathrm{C} 19)$ & 5.830 & 53. $\phi(\mathrm{C} 5-\mathrm{C} 10-\mathrm{C} 9)$ & $1.850(1.080)$ \\
\hline 8. $\nu(\mathrm{C} 4-\mathrm{C} 20)$ & 4.400 & 54. $\phi(\mathrm{C} 5-\mathrm{C} 10-\mathrm{C} 18)$ & $0.480(0.250)$ \\
\hline 9. $\nu(\mathrm{C} 5-\mathrm{C} 6)$ & 3.290 & 55. $\phi(\mathrm{C} 9-\mathrm{C} 10-\mathrm{C} 18)$ & $0.480(0.250)$ \\
\hline 10. $\nu(\mathrm{C} 5-\mathrm{C} 10)$ & 3.800 & 56. $\phi(\mathrm{C} 9-\mathrm{C} 11-\mathrm{C} 12)$ & $0.680(0.580)$ \\
\hline 11. $\nu(\mathrm{C} 6-\mathrm{C} 7)$ & 3.290 & 57. $\phi(\mathrm{C} 11-\mathrm{C} 12=\mathrm{C} 13)$ & $0.950(0.750)$ \\
\hline 12. $\nu(\mathrm{C} 7-\mathrm{C} 8)$ & 3.290 & 58. $\phi(\mathrm{C} 12=\mathrm{C} 13-\mathrm{C} 14)$ & $0.650(0.450)$ \\
\hline 13. $\nu(\mathrm{C} 8-\mathrm{C} 9)$ & 3.290 & 59. $\phi(\mathrm{C} 12=\mathrm{C} 3-\mathrm{C} 16)$ & $0.650(0.450)$ \\
\hline 14. $\nu(\mathrm{C} 8=\mathrm{C} 17)$ & 7.653 & 60. $\phi(\mathrm{C} 14-\mathrm{C} 13-\mathrm{C} 16)$ & $0.651(0.550)$ \\
\hline 15. $\nu(\mathrm{C} 9-\mathrm{C} 10)$ & 3.290 & 61. $\phi(\mathrm{C} 13-\mathrm{C} 14-\mathrm{C} 15)$ & $0.651(0.550)$ \\
\hline 16. $\nu(\mathrm{C} 9-\mathrm{C} 11)$ & 4.250 & 62. $\phi(\mathrm{C} 13-\mathrm{C} 14-\mathrm{O} 22)$ & $1.010(0.780)$ \\
\hline 17. $\nu(\mathrm{C} 10-\mathrm{C} 18)$ & 4.400 & 63. $\phi(\mathrm{C} 15-\mathrm{C} 14-\mathrm{O} 22)$ & $1.010(0.780)$ \\
\hline 18. $\nu(\mathrm{C} 11-\mathrm{C} 12)$ & 3.580 & 64. $\phi(\mathrm{C} 14-\mathrm{C} 15-\mathrm{O} 21)$ & $0.550(0.450)$ \\
\hline 19. $\nu(\mathrm{C} 12=\mathrm{C} 13)$ & 5.630 & 65. $\phi(\mathrm{C} 13-\mathrm{C} 16-\mathrm{O} 21)$ & $0.550(0.450)$ \\
\hline 20. $\nu(\mathrm{C} 13-\mathrm{C} 14)$ & 4.280 & 66. $\phi(\mathrm{C} 13-\mathrm{C} 16=\mathrm{O} 23)$ & $1.250(0.952)$ \\
\hline 21. $\nu(\mathrm{C} 13-\mathrm{C} 16)$ & 4.280 & 67. $\phi(\mathrm{C} 21-\mathrm{C} 16=23)$ & $1.550(1.050)$ \\
\hline 22. $\nu(\mathrm{C} 14-\mathrm{C} 15)$ & 4.280 & 68. $\phi(\mathrm{C} 4-\mathrm{C} 19-\mathrm{O} 24)$ & $0.550(0.350)$ \\
\hline 23. $\nu(\mathrm{C} 14-\mathrm{O} 22)$ & 4.000 & 69. $\phi(\mathrm{C} 15-\mathrm{O} 21-\mathrm{C} 16)$ & $0.412(0.350)$ \\
\hline 24. $\nu(\mathrm{C} 15-\mathrm{O} 21)$ & 6.060 & 70. $\omega(\mathrm{O} 22-\mathrm{C} 14)$ & 0.500 \\
\hline 25. $\nu(\mathrm{C} 16-\mathrm{O} 21)$ & 4.755 & 71. $\omega(\mathrm{O} 23=\mathrm{C} 16)$ & 0.380 \\
\hline 26. $\nu(\mathrm{C} 16=\mathrm{O} 23)$ & 9.239 & 72. $\omega(\mathrm{O} 25-\mathrm{C} 3)$ & 0.500 \\
\hline 27. $\nu(\mathrm{C} 19-\mathrm{O} 24)$ & 6.580 & 73. $\omega(\mathrm{C} 17=\mathrm{C} 8)$ & 0.500 \\
\hline 28. $\phi(\mathrm{C} 2-\mathrm{C} 1-\mathrm{C} 10)$ & $1.520(0.980)$ & 74. $\omega(\mathrm{C} 18-\mathrm{C} 10)$ & 0.456 \\
\hline 29. $\phi(\mathrm{C} 1-\mathrm{C} 2-\mathrm{C} 3)$ & $1.520(0.980)$ & 75. $\omega(\mathrm{C} 20-\mathrm{C} 4)$ & 0.456 \\
\hline 30. $\phi(\mathrm{C} 2-\mathrm{C} 3-\mathrm{C} 4)$ & $1.520(0.980)$ & 76. $\tau(\mathrm{C} 1-\mathrm{C} 2)$ & 0.182 \\
\hline 31. $\phi(\mathrm{C} 2-\mathrm{C} 3-\mathrm{O} 25)$ & $0.760(0.350)$ & 77. $\tau(\mathrm{C} 1-\mathrm{C} 10)$ & 0.182 \\
\hline 32. $\phi(\mathrm{C} 4-\mathrm{C} 3-\mathrm{O} 25)$ & $0.760(0.350)$ & 78. $\tau(\mathrm{C} 2-\mathrm{C} 3)$ & 0.182 \\
\hline 33. $\phi(\mathrm{C} 3-\mathrm{C} 4-\mathrm{C} 5)$ & $1.520(0.980)$ & 79. $\tau(\mathrm{C} 3-\mathrm{C} 4)$ & 0.182 \\
\hline 34. $\phi(\mathrm{C} 3-\mathrm{C} 4-\mathrm{C} 19)$ & $0.480(0.250)$ & 80. $\tau(\mathrm{C} 4-\mathrm{C} 5)$ & 0.182 \\
\hline 35. $\phi(\mathrm{C} 3-\mathrm{C} 4-\mathrm{C} 20)$ & $0.480(0.250)$ & 81. $\tau(\mathrm{C} 4-\mathrm{C} 19)$ & 0.182 \\
\hline 36. $\phi(\mathrm{C} 5-\mathrm{C} 4-\mathrm{C} 19)$ & $0.480(0.250)$ & 82. $\tau(\mathrm{C} 5-\mathrm{C} 6)$ & 0.136 \\
\hline 37. $\phi(\mathrm{C} 5-\mathrm{C} 4-\mathrm{C} 20)$ & $0.480(0.250)$ & 83. $\tau(\mathrm{C} 5-\mathrm{C} 10)$ & 0.182 \\
\hline 38. $\phi(\mathrm{C} 19-\mathrm{C} 4-\mathrm{C} 20)$ & $0.462(0.350)$ & 84. $\tau(\mathrm{C} 6-\mathrm{C} 7)$ & 0.136 \\
\hline 39. $\phi(\mathrm{C} 4-\mathrm{C} 5-\mathrm{C} 6)$ & $0.480(0.250)$ & 85. $\tau(\mathrm{C} 7-\mathrm{C} 8)$ & 0.136 \\
\hline 40. $\phi(\mathrm{C} 4-\mathrm{C} 5-\mathrm{C} 10)$ & $1.520(0.980)$ & 86. $\tau(\mathrm{C} 8-\mathrm{C} 9)$ & 0.136 \\
\hline 41. $\phi(\mathrm{C} 6-\mathrm{C} 5-\mathrm{C} 10)$ & $1.850(1.080)$ & 87. $\tau(\mathrm{C} 9-\mathrm{C} 10)$ & 0.136 \\
\hline 42. $\phi(\mathrm{C} 5-\mathrm{C} 6-\mathrm{C} 7)$ & $1.850(1.080)$ & 88. $\tau(\mathrm{C} 9-\mathrm{C} 11)$ & 0.186 \\
\hline 43. $\phi(\mathrm{C} 6-\mathrm{C} 7-\mathrm{C} 8)$ & $1.850(1.080)$ & 89. $\tau(\mathrm{C} 11-\mathrm{C} 12)$ & 0.185 \\
\hline 44. $\phi(C 7-C 8-C 9)$ & $1.850(1.080)$ & 90. $\tau(\mathrm{C} 12=\mathrm{C} 13)$ & 0.185 \\
\hline 45. $\phi(C 7-C 8=C 17)$ & $0.750(0.750)$ & 91. $\tau(\mathrm{C} 13-\mathrm{C} 14)$ & 0.181 \\
\hline \multirow[t]{4}{*}{ 46. $\phi(C 9-C 8=C 17)$} & $0.750(0.750)$ & 92. $\tau(\mathrm{C} 13-\mathrm{C} 16)$ & 0.181 \\
\hline & & 93. $\tau(\mathrm{C} 14-\mathrm{C} 15)$ & 0.181 \\
\hline & & 94. $\tau(\mathrm{C} 15-\mathrm{C} 21)$ & 0.180 \\
\hline & & 95. $\tau(\mathrm{C} 16-\mathrm{O} 21)$ & 0.060 \\
\hline
\end{tabular}

Note:

$\nu(\mathrm{X}-\mathrm{Y})$ represents the stretching between the atoms $\mathrm{X}$ and $\mathrm{Y}$ etc.

$\phi(\mathrm{X}-\mathrm{Y}-\mathrm{Z})$ represents the in plane bending between the atoms $\mathrm{X}, \mathrm{Y}$ and $\mathrm{Z}$.

$\omega(X-Y)$ represents the out of plane bending (wagging) between the atoms $X$ and $Y$.

$\tau(\mathrm{X}-\mathrm{Y})$ represents the torsion of the atoms $\mathrm{X}$ and $\mathrm{Y}$.

The number in parenthesis represents the non-bonded value of the atoms $\mathrm{X}, \mathrm{Y}$ and $\mathrm{Z}$. 
Table 2

Optimized force constant values for corresponding modes

\begin{tabular}{|c|c|c|}
\hline S. No. & Modes & Force constant value \\
\hline 1 & $\nu(\mathrm{C}-\mathrm{C}) \mathrm{R} 1$ & 3.5310 \\
\hline 2 & $\nu(\mathrm{C}-\mathrm{C}) \mathrm{R} 2$ & 3.2900 \\
\hline 3 & $\nu(\mathrm{C}-\mathrm{C}) \mathrm{R} 3$ & 4.2800 \\
\hline 4 & $\nu(\mathrm{C}-\mathrm{C}) \mathrm{COM} \mathrm{R} 1 \& \mathrm{R} 2$ & 3.8000 \\
\hline 5 & $\nu(\mathrm{C}-\mathrm{C}) \mathrm{adj}-\mathrm{R} 1$ & 4.4000 \\
\hline 6 & $\nu(\mathrm{C}=\mathrm{C}) \mathrm{adj}-\mathrm{R} 2$ & 7.6530 \\
\hline 7 & $\nu(\mathrm{C}-\mathrm{C}) \mathrm{adj}-\mathrm{R} 2$ & 4.2500 \\
\hline 8 & $\nu(\mathrm{C}=\mathrm{C}) \mathrm{adj}-\mathrm{R} 3$ & 5.6380 \\
\hline 9 & $\nu(\mathrm{C}-\mathrm{O}) \mathrm{adj}-\mathrm{R} 1$ & 4.9000 \\
\hline 10 & $\nu(\mathrm{C}=\mathrm{O}) \mathrm{adj}-\mathrm{R} 3$ & 9.2390 \\
\hline 11 & $\nu(\mathrm{C}-\mathrm{O}) \mathrm{R} 3$ & 6.0600 \\
\hline 12 & $\nu(\mathrm{C}-\mathrm{O})$ link- $\mathrm{R} 1$ & 6.5800 \\
\hline 13 & $\nu(\mathrm{C}-\mathrm{O}) \mathrm{adj}-\mathrm{R} 3$ & 4.0000 \\
\hline 14 & $\nu(\mathrm{C}-\mathrm{C}) \mathrm{BB}$ & 3.5800 \\
\hline 15 & $\nu(\mathrm{C}-\mathrm{C})$ adj- $\mathrm{R} 1-\mathrm{O}$ & 5.8300 \\
\hline 16 & $\nu(\mathrm{C}-\mathrm{O}) \mathrm{R} 3-\mathrm{adj}-\mathrm{O}$ & 4.7550 \\
\hline 17 & $\phi(\mathrm{C}-\mathrm{C}-\mathrm{C}) \mathrm{R} 1$ & 1.5200 \\
\hline 18 & $\phi(\mathrm{C}-\mathrm{C}-\mathrm{C}) \mathrm{R} 2$ & 1.8500 \\
\hline 19 & $\phi(\mathrm{C}-\mathrm{C}-\mathrm{C}) \mathrm{R} 3$ & 0.6510 \\
\hline 20 & $\phi(\mathrm{C}-\mathrm{C}-\mathrm{C}) \mathrm{adj}-\mathrm{R} 1 \& \mathrm{R} 2$ & 0.4800 \\
\hline 21 & $\phi(\mathrm{C}-\mathrm{C}-\mathrm{C}) \mathrm{BB}$ & 0.6800 \\
\hline 22 & $\phi(\mathrm{C}-\mathrm{C}=\mathrm{C}) \mathrm{adj}-\mathrm{R} 2$ & 0.7500 \\
\hline 23 & $\phi(\mathrm{C}-\mathrm{C}-\mathrm{O}) \mathrm{adj}-\mathrm{R} 3$ & 1.0100 \\
\hline 24 & $\phi(\mathrm{C}-\mathrm{C}-\mathrm{O}) \mathrm{R} 3$ & 0.5500 \\
\hline 25 & $\phi(\mathrm{O}-\mathrm{C}=\mathrm{O})$ adj-R3 & 1.5500 \\
\hline 26 & $\phi(\mathrm{C}-\mathrm{C}-\mathrm{O})$ link-R1 & 0.5500 \\
\hline 27 & $\phi(\mathrm{C}-\mathrm{O}-\mathrm{C}) \mathrm{R} 3$ & 0.4120 \\
\hline 28 & $\phi(\mathrm{C}=\mathrm{C}-\mathrm{C}) \mathrm{adj}-\mathrm{R} 3$ & 0.6500 \\
\hline 29 & $\phi(\mathrm{C}-\mathrm{C}-\mathrm{C}) \mathrm{adj}-\mathrm{R} 1$ & 0.4620 \\
\hline 30 & $\phi(\mathrm{C}-\mathrm{C}=\mathrm{O})$ adj-R3 & 1.2500 \\
\hline 31 & $\phi(\mathrm{C}-\mathrm{C}=\mathrm{C}) \mathrm{BB}$ & 0.9500 \\
\hline 32 & $\omega(\mathrm{O}-\mathrm{C}) \mathrm{R} 1 \& \mathrm{R} 3$ & 0.5000 \\
\hline 33 & $\omega(\mathrm{O}=\mathrm{C}) \mathrm{R} 3$ & 0.3800 \\
\hline 34 & $\omega(\mathrm{C}=\mathrm{C}) \mathrm{R} 2$ & 0.5000 \\
\hline 35 & $\omega(\mathrm{C}-\mathrm{C}) \mathrm{R} 1$ & 0.4560 \\
\hline 36 & $\tau(\mathrm{C}-\mathrm{C}) \mathrm{R} 1$ & 0.1820 \\
\hline 37 & $\tau(\mathrm{C}-\mathrm{C})$ adj-R1-O & 0.1820 \\
\hline 38 & $\tau(\mathrm{C}-\mathrm{C}) \mathrm{R} 2$ & 0.1356 \\
\hline 39 & $\tau(\mathrm{C}-\mathrm{C})$ common $\mathrm{R} 1 \& \mathrm{R} 2$ & 0.1820 \\
\hline 40 & $\tau(\mathrm{C}-\mathrm{C}) \mathrm{adj}-\mathrm{R} 2$ & 0.1856 \\
\hline 41 & $\tau(\mathrm{C}-\mathrm{C}) \mathrm{BB}$ & 0.1850 \\
\hline 42 & $\tau(\mathrm{C}=\mathrm{C}) \mathrm{adj}-\mathrm{R} 3$ & 0.1850 \\
\hline 43 & $\tau(\mathrm{C}-\mathrm{C}) \mathrm{R} 3$ & 0.1810 \\
\hline 44 & $\tau(\mathrm{C}-\mathrm{O}) \mathrm{R} 3$ & 0.1800 \\
\hline 45 & $\tau(\mathrm{C}-\mathrm{O}) \mathrm{R} 3-$ adj-O & 0.0600 \\
\hline
\end{tabular}

Note: Abbreviations used in the table have following meanings: R - Ring; Adj - Adjacent to; BB - Back Bone.

optimized force constant values for the corresponding modes are given in Table 2. The frequencies along with the potential energy distribution are given in Table 3. The FT-IR spectra of Andrographolide is given in Fig. 2. The observed frequencies agree with the calculated ones within $5 \mathrm{~cm}^{-1}$. In the assignment of the normal modes only the dominant potential energy distributions are given. Identification with 
Table 3

Calculated and observed vibrational modes of andrographolide

\begin{tabular}{|c|c|c|}
\hline $\begin{array}{l}\text { Calculated freq. } \\
\mathrm{cm}^{-1}\end{array}$ & $\begin{array}{l}\text { Observed freq. } \\
\mathrm{cm}^{-1}\end{array}$ & Assignment (\% Potential energy distribution) \\
\hline 1728 & 1728 & $\nu(\mathrm{C}=\mathrm{O}) \mathrm{adj}-\mathrm{R} 3[77]+\nu(\mathrm{C}-\mathrm{C}) \mathrm{R} 3[18]+\nu(\mathrm{C}-\mathrm{O}) \mathrm{R} 3 \mathrm{adj}-\mathrm{O}[6]+\phi(\mathrm{C}-\mathrm{C}-\mathrm{O}) \mathrm{R} 3[6]$ \\
\hline 1675 & 1675 & $\nu(\mathrm{C}=\mathrm{C}) \operatorname{adj}-\mathrm{R} 2[67]+\phi(\mathrm{C}-\mathrm{C}-\mathrm{C}) \mathrm{R} 2[26]+\nu(\mathrm{C}-\mathrm{C}) \mathrm{R} 2[12]$ \\
\hline 1596 & 1595 & $\begin{array}{l}\nu(\mathrm{C}=\mathrm{C}) \operatorname{adj}-\mathrm{R} 3[47]+\nu(\mathrm{C}-\mathrm{C}) \mathrm{R} 3[34]+\phi(\mathrm{C}-\mathrm{C}=\mathrm{O}) \operatorname{adj}-\mathrm{R} 3[11] \\
\quad+\nu(\mathrm{C}-\mathrm{O}) \mathrm{R} 3 \text { adj-O [9] }+\phi(\mathrm{C}-\mathrm{C}-\mathrm{C}) \mathrm{R} 3[8]+\phi(\mathrm{O}-\mathrm{C}=\mathrm{O}) \operatorname{adj}-\mathrm{R} 3[6]\end{array}$ \\
\hline 1455 & 1459 & $\begin{array}{l}\nu(\mathrm{C}-\mathrm{C}) \text { com R1\&R2 [44] }+\phi(\mathrm{C}-\mathrm{C}-\mathrm{C}) \mathrm{R} 2[16] \\
\quad+\phi(\mathrm{C}-\mathrm{C}-\mathrm{C}) \mathrm{R} 1[15] \nu(\mathrm{C}-\mathrm{C}) \mathrm{R} 2[13]+\nu(\mathrm{C}-\mathrm{C}) \text { adj-R1 [10] } \\
\quad+\nu(\mathrm{C}-\mathrm{C}) \text { adj-R2 [10] } \phi(\mathrm{C}-\mathrm{C}-\mathrm{C}) \mathrm{adj}-\mathrm{R} 1 \& \mathrm{R} 2[7]\end{array}$ \\
\hline 1416 & 1420 & $\begin{array}{l}\nu(\mathrm{C}-\mathrm{C}) \text { adj-R1-O [33] }+\nu(\mathrm{C}-\mathrm{C}) \mathrm{adj}-\mathrm{R} 1[19]+\phi(\mathrm{C}-\mathrm{C}-\mathrm{C}) \mathrm{R} 1[10] \\
\quad+\nu(\mathrm{C}-\mathrm{C})-\mathrm{R} 1[9]+\phi(\mathrm{C}-\mathrm{C}-\mathrm{C}) \mathrm{R} 2[8]+\phi(\mathrm{C}-\mathrm{C}-\mathrm{C}) \text { adj-R1\&R2 [6] } \\
\quad+\nu(\mathrm{C}-\mathrm{O}) \text { link}-\mathrm{R} 1[6]\end{array}$ \\
\hline 1367 & 1367 & $\begin{array}{l}\nu(\mathrm{C}-\mathrm{C}) \mathrm{R} 1[22]+\nu(\mathrm{C}-\mathrm{O}) \text { adj-R1 [22] }+\nu(\mathrm{C}-\mathrm{C}) \mathrm{R} 2[16]+\phi(\mathrm{C}-\mathrm{C}-\mathrm{C}) \mathrm{R} 1[15] \\
\nu(\mathrm{C}-\mathrm{C}) \text { adj-R2 [11] }+\nu(\mathrm{C}-\mathrm{C}) \text { adj-R1 [7] }+\phi(\mathrm{C}-\mathrm{C}-\mathrm{C}) \mathrm{R} 2[7]\end{array}$ \\
\hline 1299 & 1297 & $\begin{array}{l}\nu(\mathrm{C}-\mathrm{O}) \mathrm{R} 3[25]+\nu(\mathrm{C}-\mathrm{C}) \mathrm{R} 3[23]+\phi(\mathrm{O}-\mathrm{C}=\mathrm{O}) \mathrm{adj}-\mathrm{R} 3[11] \\
\quad+\phi(\mathrm{C}=\mathrm{C}-\mathrm{C}) \mathrm{adj}-\mathrm{R} 3[10]+\nu(\mathrm{C}=\mathrm{C}) \mathrm{adj}-\mathrm{R} 3[8]+\nu(\mathrm{C}-\mathrm{C}) \mathrm{BB}[8]\end{array}$ \\
\hline 1262 & 1266 & $\begin{array}{l}\nu(\mathrm{C}-\mathrm{O}) \mathrm{R} 3[46]+\nu(\mathrm{C}-\mathrm{C}) \mathrm{R} 3[20]+\nu(\mathrm{C}-\mathrm{O}) \mathrm{R} 3 \text { adj-O }[10] \\
\quad+\nu(\mathrm{C}=\mathrm{C}) \text { adj-R3 }[9]+\nu(\mathrm{C}-\mathrm{C}) \mathrm{BB}[8]\end{array}$ \\
\hline 1222 & 1220 & $\begin{array}{l}\nu(\mathrm{C}-\mathrm{O}) \text { link-R1 [31] }+\nu(\mathrm{C}-\mathrm{C}) \mathrm{R} 2[30]+\phi(\mathrm{C}-\mathrm{C}-\mathrm{C}) \mathrm{R} 2[15] \\
\quad+\nu(\mathrm{C}-\mathrm{C}) \operatorname{adj}-\mathrm{R} 1[11]\end{array}$ \\
\hline 1157 & 1158 & $\nu(\mathrm{C}-\mathrm{C}) \mathrm{R} 3[60]+\nu(\mathrm{C}-\mathrm{O}) \mathrm{R} 3[16]+\nu(\mathrm{C}-\mathrm{O}) \operatorname{adj}-\mathrm{R} 3[13]+\phi(\mathrm{C}-\mathrm{C}-\mathrm{O}) \operatorname{adj}-\mathrm{R} 3[9]$ \\
\hline 1085 & 1079 & $\begin{array}{l}\nu(\mathrm{C}-\mathrm{O}) \operatorname{adj}-\mathrm{R} 3[51]+\phi(\mathrm{C}-\mathrm{C}-\mathrm{O}) \operatorname{adj}-\mathrm{R} 3[14]+\nu(\mathrm{C}-\mathrm{C}) \mathrm{R} 1[13] \\
\quad+\nu(\mathrm{C}-\mathrm{C}) \mathrm{R} 1[9]\end{array}$ \\
\hline 1031 & 1034 & $\nu(\mathrm{C}-\mathrm{C}) \mathrm{BB}[51]+\nu(\mathrm{C}-\mathrm{C}) \mathrm{R} 1[10]+\nu(\mathrm{C}-\mathrm{C}) \mathrm{R} 2[9]+\nu(\mathrm{C}-\mathrm{O}) \mathrm{R} 3 \mathrm{adj}-\mathrm{O}[7]$ \\
\hline 974 & 981 & $\begin{array}{l}\phi(\mathrm{C}-\mathrm{C}-\mathrm{C}) \mathrm{R} 2[28]+\nu(\mathrm{C}-\mathrm{C}) \mathrm{R} 2[20]+\nu(\mathrm{C}-\mathrm{C}) \mathrm{R} 1[10]+\nu(\mathrm{C}-\mathrm{C}) \mathrm{adj}-\mathrm{R} 2[9] \\
\quad+\omega(\mathrm{C}=\mathrm{C}) \mathrm{R} 2[9]+\nu(\mathrm{C}-\mathrm{C}) \mathrm{BB}[8]\end{array}$ \\
\hline 907 & 909 & $\begin{array}{l}\phi(\mathrm{C}-\mathrm{C}-\mathrm{C}) \mathrm{R} 1[12]+\nu(\mathrm{C}-\mathrm{C}) \mathrm{R} 2[12]+\nu(\mathrm{C}-\mathrm{C}) \mathrm{R} 1[8]+\phi(\mathrm{C}-\mathrm{C}-\mathrm{O}) \operatorname{adj}-\mathrm{R} 3[8] \\
\quad+\nu(\mathrm{C}-\mathrm{C}) \mathrm{R} 3[7]+\nu(\mathrm{C}-\mathrm{C}) \text { adj-R1 [6] }\end{array}$ \\
\hline 877 & 875 & $\begin{array}{l}\phi(\mathrm{C}-\mathrm{C}-\mathrm{C}) \mathrm{R} 2[28]+\nu(\mathrm{C}-\mathrm{C}) \text { adj-R } 1[18]+\nu(\mathrm{C}-\mathrm{C}) \mathrm{R} 2[10] \\
\quad+\nu(\mathrm{C}-\mathrm{C}) \mathrm{com}-\mathrm{R} 1 \& \mathrm{R} 2[10]+\phi(\mathrm{C}-\mathrm{C}-\mathrm{C}) \mathrm{R} 1[7]+\nu(\mathrm{C}-\mathrm{C}) \mathrm{R} 1[6]\end{array}$ \\
\hline 817 & 816 & $\begin{array}{l}\tau(\mathrm{C}-\mathrm{C}) \mathrm{R} 3[18]+\tau(\mathrm{C}=\mathrm{C}) \mathrm{adj}-\mathrm{R} 3[11]+\nu(\mathrm{C}-\mathrm{O}) \mathrm{adj}-\mathrm{R} 3[10]+\phi(\mathrm{C}-\mathrm{C}=\mathrm{C}) \mathrm{BB}[8] \\
\quad+\omega(\mathrm{O}=\mathrm{C}) \mathrm{R} 3[7]+\nu(\mathrm{C}-\mathrm{C}) \mathrm{R} 3[7]+\phi(\mathrm{C}-\mathrm{C}-\mathrm{C}) \mathrm{BB}[6]\end{array}$ \\
\hline 708 & 715 & $\begin{array}{l}\phi(\mathrm{C}-\mathrm{C}-\mathrm{C}) \mathrm{R} 2[12]+\phi(\mathrm{C}-\mathrm{C}-\mathrm{C}) \mathrm{adj}-\mathrm{R} 1 \& \mathrm{R} 2[10]+\phi(\mathrm{C}-\mathrm{C}-\mathrm{C}) \mathrm{R} 12[10] \\
\quad+\nu(\mathrm{C}-\mathrm{O}) \mathrm{R} 3 \mathrm{adj}-\mathrm{O}[8]+\tau(\mathrm{C}-\mathrm{C}) \mathrm{R} 3[6]+\omega(\mathrm{O}-\mathrm{C}) \mathrm{R} 1 \& \mathrm{R} 3[5]\end{array}$ \\
\hline 659 & 659 & $\begin{array}{l}\phi(\mathrm{C}-\mathrm{C}=\mathrm{C}) \operatorname{adj}-\mathrm{R} 2[30]+\phi(\mathrm{C}-\mathrm{C}-\mathrm{C}) \mathrm{R} 2[16]+\phi(\mathrm{C}-\mathrm{C}-\mathrm{C}) \text { adj-R1\&R2 [7] } \\
\quad+\phi(\mathrm{C}-\mathrm{C}-\mathrm{C}) \mathrm{R} 1[7]+\nu(\mathrm{C}-\mathrm{C}) \mathrm{R} 1[7]\end{array}$ \\
\hline 576 & 572 & $\begin{array}{l}\omega(\mathrm{O}=\mathrm{C}) \mathrm{R} 3[20]+\phi(\mathrm{C}-\mathrm{C}-\mathrm{O}) \mathrm{R} 3[15]+\phi(\mathrm{C}-\mathrm{C}-\mathrm{C}) \mathrm{R} 3[14]+\phi(\mathrm{C}-\mathrm{O}-\mathrm{C}) \mathrm{R} 3[12] \\
\quad+\tau(\mathrm{C}-\mathrm{O}) \mathrm{R} 3[7]+\tau(\mathrm{C}-\mathrm{C}) \mathrm{R} 3[6]\end{array}$ \\
\hline
\end{tabular}

Note: Abbreviations used in the table have following meanings: R - Ring; Adj - Adjacent to; Com - Common to; BB Back Bone.

the experimental data has been made on the basis of potential energy distribution, line profile, relative intensities, energies and the presence/absence of a given mode in similar molecules.

Although a number of spectroscopic studies [30-33] have been performed on andrographis paniculata but presumably, the data for the normal mode analysis on andrographolide is being reported for the first time and this makes it imperative to discuss all the vibrational modes with significant potential energy distribution and hence their relation with the conformation of andrographolide (Fig. 1). All the significant vibrational modes involving the prime sites are discussed as follows: 


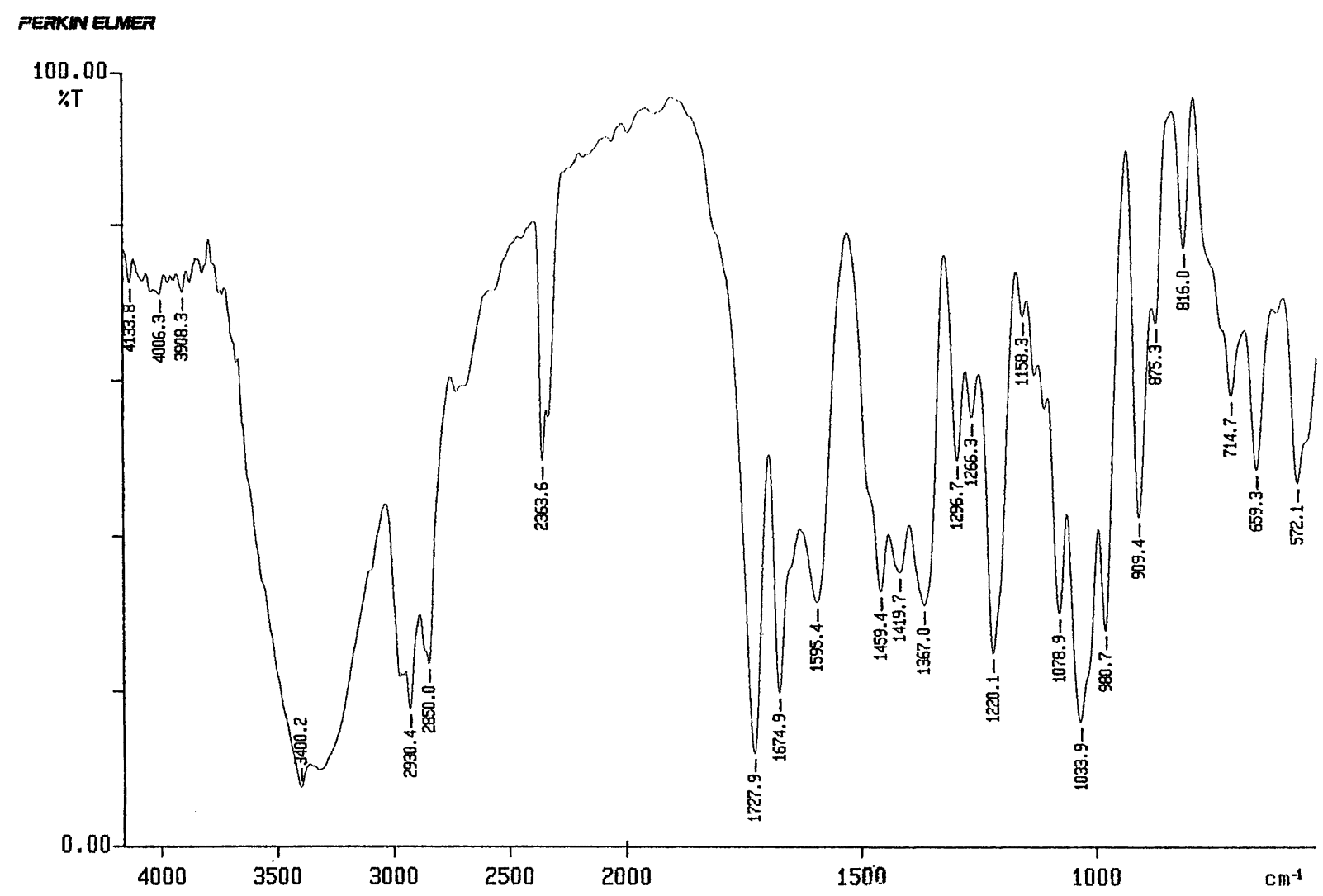

Fig. 2. FT-IR spectra of Andrographolide (4000-550) $\mathrm{cm}^{-1}$.

\subsection{Rings $R 1, R 2, R 3$}

The molecule under study is a diterpene which has $\mathrm{C} 4$ primary alcoholic group $(\mathbf{C H 2}-\mathbf{O H})$ and this furan ring assumes importance because of the bioactivity point of view. As per the normal mode calculations, the stretching mode appearing at $1728 \mathrm{~cm}^{-1}$ has a significant potential energy distribution (P.E.D) of $(\mathrm{C} 16=\mathrm{O} 23) 77 \%$, that can be possibly explained by the fact that $\mathrm{C} 16$ carbonyl atom is in conjugation with $\mathrm{C} 12=\mathrm{C} 13$ on one end and ring $\mathrm{O} 21$ on the other hand. This conjugation imparts negative charge to the $\mathrm{C}=\mathrm{O}$ carbon by delocalisation. The above observation can also compared with $\mathrm{C}=\mathrm{O}$ stretching mode in case of gamma-butyrolactone [34] in which it appears at $1772 \mathrm{~cm}^{-1}$. This value is very typical for similar modes in lactones or similar fragments [35]. The band at $572 \mathrm{~cm}^{-1}$ was assigned to the in-plane and out-of-plane deformations, respectively, of the carbonyl group. These assignments are based on the facts that carbonyl deformation modes do not normally give strong absorptions and occur in this general region.

As suggested by the crystallographic study and along with our normal mode calculations it can be said that the ring $\mathrm{R} 3$ remains in puckered form and R1, R2 in chair form, which is further, evidenced by a high percentage of potential energy distribution of (C12-C13) $47 \%$ and (C8-C17) 67\%. The potential energy distribution data further suggested that $\mathrm{C}$ attached to the heteroatom i.e., $\mathrm{O}$ has high p.e.d. Value e.g. (C15-O21) 46\%. The low value of (C3-O25) 22\% as compared to (C14-O22) 51\% is suggestive of the close proximity of $\mathrm{C} 14-\mathrm{O} 22$ by ring junction (C12-C11) $51 \%$ and rings $\mathrm{R} 1$ and $\mathrm{R} 2$. For the vibrational 
band at $659 \mathrm{~cm}^{-1}$ the normal mode calculation yields a potential energy distribution with $\phi(\mathrm{C}-\mathrm{C}=\mathrm{C})$ $30 \%$ whereas the deformation $\phi(\mathrm{C}-\mathrm{C}-\mathrm{C})$ is only $16 \%$. A lot of similarity is found between the normal mode analysis and the $\mathrm{nmr}$ study [30,31] performed on andrographolide. The downfield signal for $\mathrm{H}$ (12) was used as starting point. A decoupling experiment established couplings of $6.8 \mathrm{~Hz}$ to the proton at $2.63 \mathrm{ppm}(\mathrm{H}(11 \mathrm{a}))$.

And $2.58 \mathrm{ppm}(\mathrm{H}(11 \mathrm{~b}))$. Although these protons overlap somewhat, the vicinal couplings to $\mathrm{H}(9 \mathrm{a})$ $(1.92 \mathrm{ppm})$ of $3.9 \mathrm{~Hz}$ for $\mathrm{H}(11 \mathrm{a})$ and $\mathrm{H}(11 \mathrm{~b})(16.5 \mathrm{~Hz})$ has been observed previously for similar systems and was attributed to the hyperconjugative effect of the vinyl group. This is also supported by the potential energy distribution of (C12-C11) 51\%.

\section{Conclusions}

The aim of this work was to characterize the dynamics of the diterpene andrographis paniculata and based on the biochemical, spectroscopic and the present study it can be said that the plant Andrographis paniculata offers an inspiring promise in many areas of medicinal importance and requires more clinical investigation along with basic research. The normal mode analysis of andrographolide shows a dynamical behaviour and possibly, opens up an avenue for further conformational research. And lastly, receptor binding action needs to be investigated thoroughly which probably can be explained by ab initio and DFT methods and this is the subject of proposed study on andrographolide which shall be reported shortly.

The author wishes to acknowledge the support of Dr. Kanwal Raj, Medicinal and Process Chemistry Division, CDRI, Lucknow, India for the experimental and spectroscopic data on andrographolide including the sample. The author also wishes to acknowledge the help received from Prof. Sudha Jain, Chemistry Department, Lucknow University for meaningful suggestions.

\section{References}

[1] A.K. Nadkarni and K.M. Nadkarni, Indian Materia Medica, Bombay Popular Prakashan 1 (1976), 101-103.

[2] D. Bensky, A. Gamble and T. Kaptchuk, Chinese Herbal Medicine Materia Medica, Revised Ed., Seattle Eastland press, $1993,95$.

[3] K. Bone, "The Story of Andrographis paniculata, a new immune system" a herb, Nutrition and Healing (1998), Sept. $3,4,8,9$ [review].

[4] A. Leelarasamee, A.S. Trakulsomboon and N. Sittisomoong, Undetectable antibacterial activity of andrographis paniculata (Burma) Wall.ex ness, J. Med. Assoc. Thail. 73 (1990), 299-304.

[5] A. Plubrukarn, S. Pinsuwan, S. Ingkatawornwong and T. Supavita, Stability of andrographolide in powdered andrographis herb under accelerated conditions, PLANTA MEDICA 72 (2006), 954-956.

[6] S.R. Jada, A.S. Hamzah, N.H. Lajis, M.S. Saad, M.F. Stevens and J. Stanlas, Semisynthesis and cytotoxic activities of andrographolide analogues, J. Enzyme Inhib. Med. Chem. 21(2) (2006), 145-155.

[7] J.R. Hanson, Diterpenoids, Nat. Prod. Rep. 21 (2004), 785-793.

[8] N. Pholphana, N. Rangkadilok, S. Thongnese, S. Ruchirawat, M. Ruchirawat and J. Satyavivad, Determination and variation of three active diterpenoids in Andrographis paniculata (Burm.f) Nees, Phytochem. Anal. 15(6) (2004), 365-371.

[9] L. Lomlim, N. Jirayupong and A. Plubrukarn, Heat accelerated degradation of solid-state Andrographolide, Chem. Pharm. Bull. 51(1) (2003), 24-26.

[10] N. Misra, P. Tandon, V.D. Gupta and V.P. Gupta, Dynamics of $\mathrm{Met}^{5}$ and $\mathrm{Leu}^{5}$ enkephalin and their receptor binding activity in relation to morphine, Indian J. Biochem. Biophys. 31 (1994), 43-54.

[11] N. Misra, P. Tandon, V.D. Gupta, V.P. Gupta and D.C. Gupta, Recent advances in the study of neuro transmitter receptors, edited by R.S. Rapaka, Drug Research Perspectives, CDRI publication, 17 (1994), 403-417.

[12] N. Misra, O. Prasad, L. Sinha, G.P. Gupta, R.C. Agnihotri and J.N. Lal, Theoretical study of temperature induced phase transitions in poly( $\beta$-benzyl-L-aspartate) and its copolymer, Polymer 46 (2005), 7450-7455. 
[13] N. Misra, O. Prasad, L. Sinha, G.P. Gupta and R.C. Agnihiotri, Pressure induced order-disorder transition in a diblock copolymer, Polymer 46 (2005), 11876-11880.

[14] N. Misra, O. Prasad, L. Sinha, G.P. Gupta, C. Mehrotra, R.C. Agnihotri and J.N. Lal, Theoretical study of temperature induced transition and hyper stability of collagen mimics, Polymers 47 (2006), 1674-1677.

[15] N. Misra, O. Prasad and L. Sinha, Vibrational dynamics of morphine in relation to Leu ${ }^{5}$ and $\mathrm{Met}^{5}$ enkaphalins, Indian Journ. Biochem. Biophys. 43 (2006), 173-181.

[16] N. Misra, O. Prasad and L. Sinha, 2006, communicated to Biophysics (Japan).

[17] N. Misra, O. Prasad and L. Sinha, 2006, communicated to Theochem.

[18] M.T. Vijayvitthal, S. Kumar and K. Raj, Oxidation studies in Andrographolide, Nat. Prod. Res. (2006), Accepted (Manuscript No NPR-1-210758).

[19] E.B.Jr. Wilson, J.C. Decius and P.C. Cross, Molecular Vibrations, Dover Publications, New York, 1980, pp. $208-239$.

[20] E.B.Jr. Wilson, A method of obtaining the expanded secular equation for the vibration frequencies of a molecule, Chem. Phys. 7 (1939), 1047-1052.

[21] E.B.Jr. Wilson, Some mathematical methods for the study of molecular vibrations, J. Chem. Phys. 9 (1941), 76-78.

[22] H.C. Urey, C.A. Bradley, The vibrations of pentatonic tetrahedral molecules, Phys. Rev. 38 (1931), 1969-1978.

[23] S. Gupta, M.A. Chaudhary, J.N.S. Yadav, V. Srivastava and J.S. Tandon, Anti-diarrheal activity of diterpenes of andrographis paniculata (Kal-megh) against Escheritia coli enterotoxin in in vivo models, Int. J. Crud. Drug. Res. (1990), 273.

[24] C. Hu, B. Zhao and Z. Caoyo, Spectrometric identification of dierpene-andrographolide, CA (1981), 531, 9720702m.

[25] T. Fujita, R. Fujitani, Y. Takeda, Y. Takashi, T. Yamada, M. Kido and I. Minza, X-ray studies on andrographolide, Chem. Parm. Bull. 32 (1984), 2117-2125.

[26] A. Basak, S. Cooper, A.G. Roberge, U.K. Banik, M. Chretiens and N.G. Seidah, Inhibition of Proprotein convertases-1,7 and furin by diterpenes of Andrographis Paniculata and their succinoyl esters, Biochem. J. 338 (1999), 107-113.

[27] P.L. Smith, K.N. Maloney, R.G. Pothen, J. Clardy and D.E. Clapham, Bisandro from AP activates TRPV4 Channels, J. Biol. Chem. 281 (2006), 29897-29904.

[28] T. Shimanouchi, Computer Program for Normal Coordinate Treatment of Polyatomic Molecules, University of Tokyo, 1968.

[29] A.L. Spek, A.M.J. Duisenberg and R.P. Labadie, Hydrogen bonding in Andrographolide, Acta Cryst. (1987), 530-532.

[30] D. Zhao, K. Liao, X. Ma and X. Yan, Study of the supramolecular Inclusion of $\beta$-Cyclodextrin with andrographolide, J. Inclusion. Phenomenon 43 (2002), 259-264.

[31] H.W. Xu, J. Zhang, H.M. Liu and J.F. Wang, Synthesis of andrographolide cyclophosphate derivatives and their antitumour activities, Synthetic Communications 36 (2006), 407-414.

[32] Y. Xinsheng, L. Xiangmin, H. Ke, Q. Feng, G. Hao, L. Jiankuan and H. Xiangjiu, Identification of a rare sulphonic acid metabolite of andrographolide in rats, DMD 31 (2003), 983-985.

[33] A.B. Smith, B.H. Toder, P.J. Carroll and J. Donohue, Stereochemistry of andrographolide, J. Cryst. Spectrosc. Res. 12(4) (1982), 309-319.

[34] D.P. Mcdermott, Vibrational assignments and normal coordinate analyses of gamma-butyrlactone and 2-pyrrolodinones, J. Phys. Chem. 90 (1986), 2569-2576.

[35] R.M. Silverstein, G.C. Bassler and T.C. Morrill, Spectrometric Identification of Organic Compounds, 4th edn, Wiley, New York, 1981. 


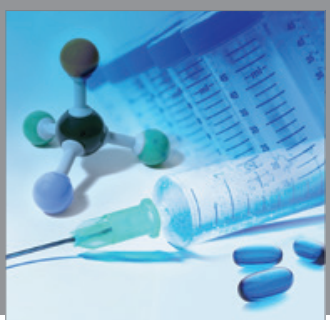

International Journal of

Medicinal Chemistry

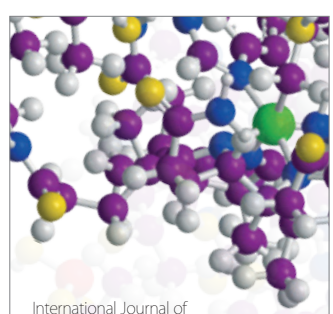

Carbohydrate Chemistry

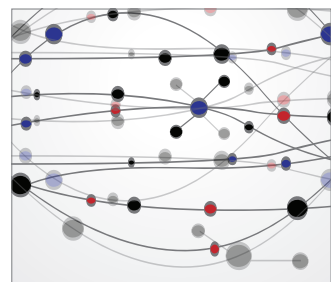

The Scientific World Journal
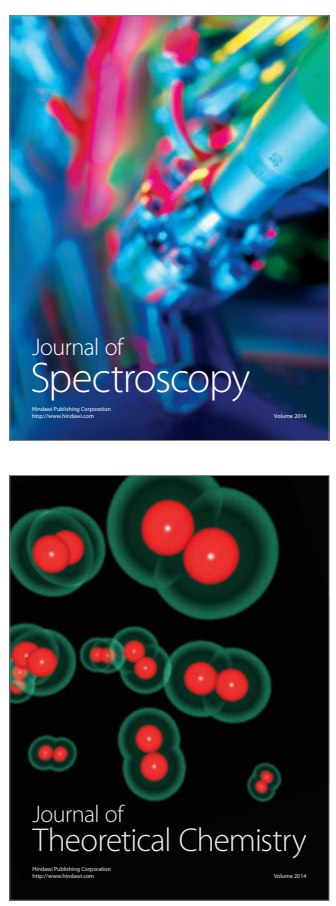
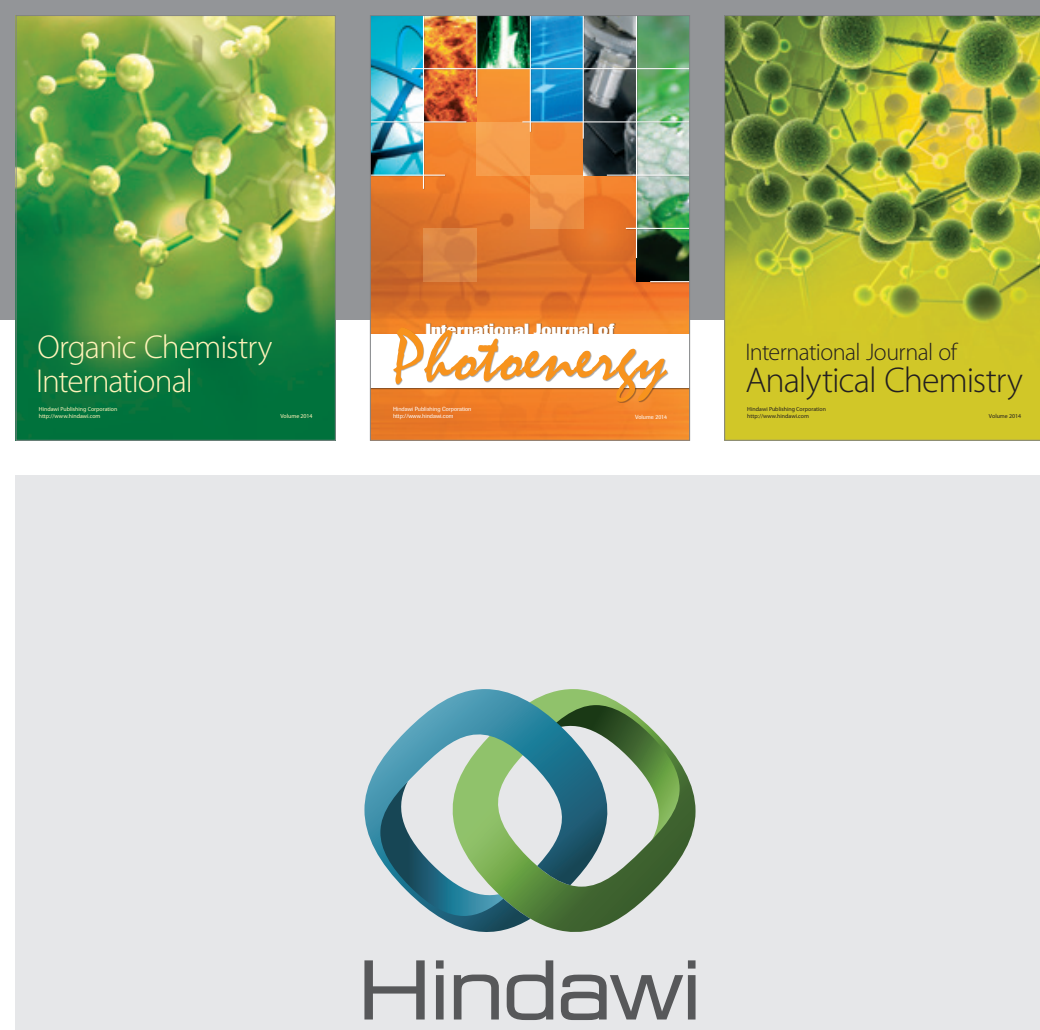

Submit your manuscripts at

http://www.hindawi.com
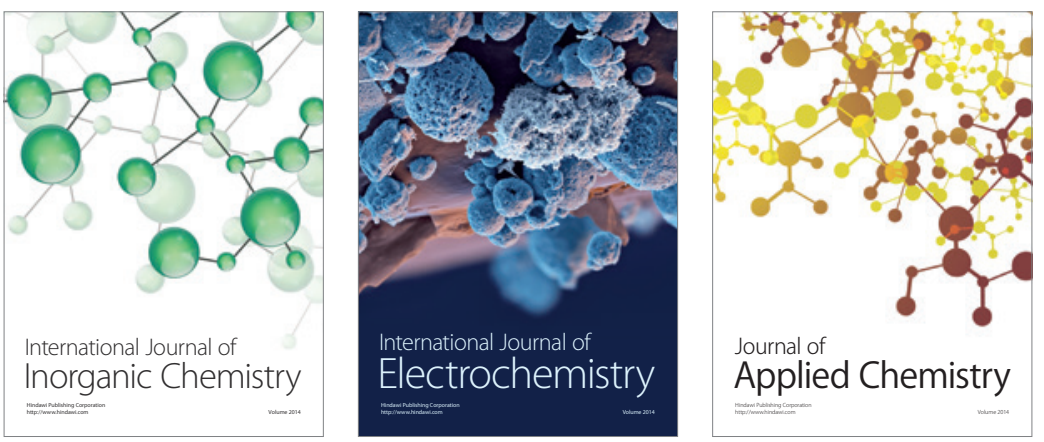

Journal of

Applied Chemistry
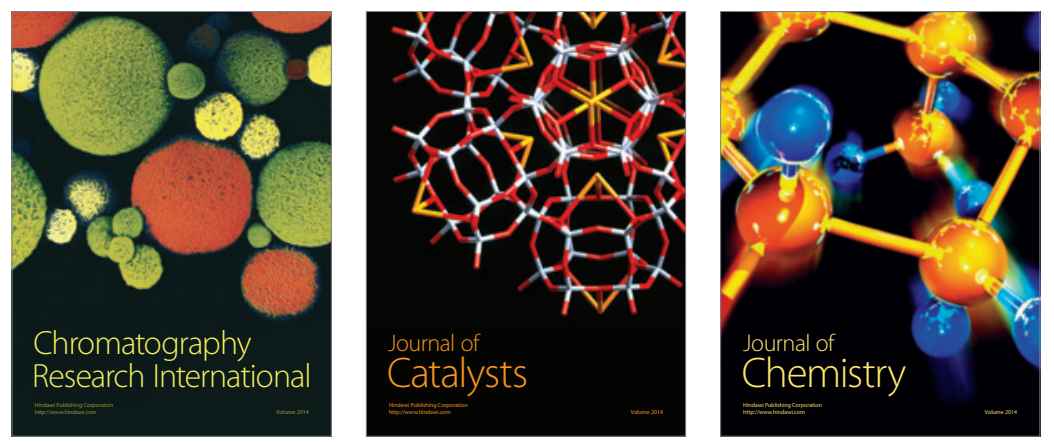
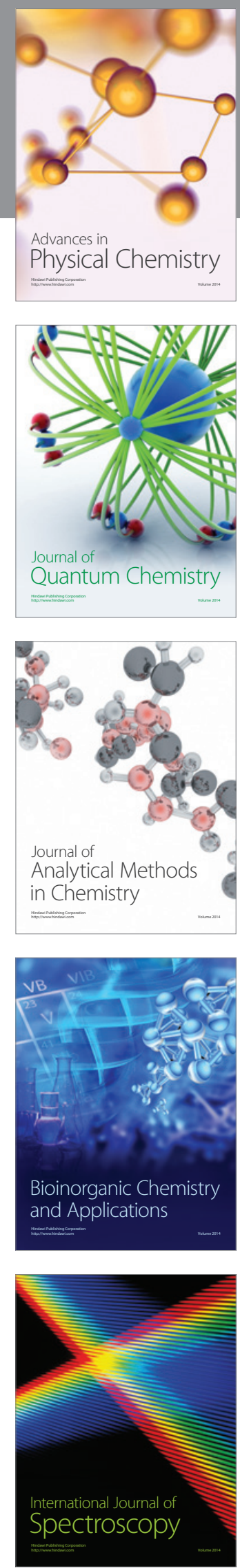